\title{
Patient-Controlled Epidural Analgesia
}

National Cancer Institute

\section{Source}

National Cancer Institute. Patient-Controlled Epidural Analgesia. NCI Thesaurus. Code C123385.

A method of analgesia that uses an epidural catheter and a pump to allow the patient to administer predetermined doses of analgesic into the epidural space as needed to maintain adequate pain control. 